qualified doctors assessed a patient with her distressed husband, commenced initial treatment and presented their case on the subsequent consultant post-take ward round. Training and orientation on local palliative care guidelines and resources followed. The benefit of this was assessed by anonymous questionnaire completed once the doctors had been in post for a month. They rated confidence in communication skills, managing symptoms, prescribing, decision making and holistic care approach.

Results $73 \%$ graded the exercise as highly beneficial. FY1 doctors confidence in the areas mentioned above ranged from 6.6 to 7.6 on a scale of $0-10$. The weakest area was discussing DNAR with patients, while the strongest area was confidence with end of life medications and doses. Other low scoring areas all focused around communicating either with the patient or their family, and making decisions about appropriate care (6.8-6.9). Areas of confidence focused around clinical aspects of care including managing symptoms, medications and assessing patient's needs (7.0-7.4).

Conclusion FY1s find communication skills and difficult conversations challenging. There is room for improvement across all areas analysed in order to achieve high confidence levels. We will continue to address these with two further high fidelity simulation sessions which will be assessed for benefit and improvement against the baseline confidence levels. These results will also be presented. Ultimately our aim is to help the new doctors achieve their competencies and provide high quality holistic care.

\section{SMALL GROUP RESEARCH IN THE EAST OF ENGLAND: A NOVEL APPROACH TO PALLIATIVE MEDICINE RESEARCH TRAINING}

Chloe Chin, Sarah Grove, Sarah Treaddell, Stephen Barclay, Anna Spathis. University of Cambridge, Cambridge University Hospitals NHS Foundation Trust, Arthur Rank Hospice, Garden House Hospice, Sue Ryder Thorpe Hall Hospice

\subsection{6/bmjspcare-2018-ASPabstracts.47}

Background Research is essential to advance the evidence base of Palliative Medicine. Few clinical trainees have the opportunity to become involved in research studies or to receive research training, which hampers trainees' ability to attain research competencies. Attending research courses may improve knowledge about research but are no substitute for participating in a study. Rotation of trainees also hinders completion of projects once started.

Aim To improve clinical trainee involvement in research to facilitate achievement of research competencies.

Development of novel approach A UK-wide survey of research training was undertaken. It found that over $75 \%$ of regions had no regular research training available. Only one region had a research training programme available for all Palliative Medicine trainees.

A programme was devised whereby East of England trainees were organised pragmatically by job location into a small research groups. Four out of twelve monthly study days were dedicated to research projects and training. Two clinical academic Palliative Medicine consultants provided supervision and met with all project groups twice yearly for advice and guidance.
Outcomes Since programme inception in 2015, outputs have included two poster and one oral presentations at national and international conferences, and one peer-reviewed journal publication. Three further projects are ongoing. Prior to the programme, research outputs were rare.

Participants report that project completion was facilitated by sharing workload amongst trainees, and by keeping projects location-based with newly rotating trainees providing continuity. The regional research meetings provided peer-to-peer support, the groups learning from each other's experiences.

Conclusion This novel approach has enhanced trainee research skills, experience and outputs. This form of research training programme could be readily adopted across the UK, being largely trainee-led: only intermittent support from consultants with an interest in research is needed.

\section{REDUCING HOSPICE ACQUIRED PRESSURE ULCERS: A THREE YEAR INITIATIVE}

Diana Sketchley. Weldmar Hospice Care, Joseph Weld Hospice

\subsection{6/bmjspcare-2018-ASPabstracts.48}

This poster outlines key implementations taken at Joseph Weld Hospice to reduce Hospice acquired pressure ulcers. Key areas for improvement were highlighted and improvements were made in assessment skills, documentation, education, Nurse/Doctor/Patient communication, reporting and hospice equipment.

Nursing assessment improvements were made through education including power point presentations: classification, risk factors and prevention. A pocket size pressure ulcer classification tool was designed for staff to have as an easy reference at the patient's bedside.

Documentation improvements included a redesign of the assessment windows on our electronic patient records to prompt accurate documentation.

Communication improvements in highlighting patients at high risk through a more detailed handover ensuring reports included any patients at risk of developing pressure ulcers.

Equipment was re-evaluated with patients need being assessed and utilised for our high risk patients and if in use air mattresses were sourced for hire to ensure pressure ulcer prevention. In May 2017 the hospice held a 'Comfort Appeal' to enable the hospice to fund new air mattresses. This exceeded our expectations raising funds within 2 months to buy 12 mattresses.

Results Year one, April 2014/2015 22 Patients developed pressure ulcers with 10 Pressure Ulcers reported as AVOIDABLE.

Year Two, April 2015/2016 23 Patients developed pressure ulcers but there was a huge reduction in AVOIDABLE pressure ulcers with only 2 reported.

Year Three, April 2016/2017 15 patients developed pressure ulcers but only 2 AVOIDABLE pressure ulcer reported.

Conclusion In making these key improvements there have been a significant reduction in hospice acquired pressure ulcers and remarkably the reduction in AVOIDABLE pressure Ulcers. The future hope for the hospice is to continue our best practice in minimising acquired pressure ulcers and abolish AVOIDABLE pressure ulcers. 